\title{
Modeling solid-solid phase transformations: from single crystal to polycrystal behavior
}

\author{
R.C. AlBERS, R. AHLUWALIA, T. LOOKMAN and A. SAXENA \\ Theoretical Division, Los Alamos National Lab, Los Alamos, New Mexico 87545 USA \\ E-mails: rca@lanl.gov / rajeev@lanl.gov / txl@lanl.gov / avadh@lanl.gov
}

\begin{abstract}
We introduce a framework for modeling elastic properties of shape memory alloy polycrystals by coupling orientational degrees of freedom with elastic strains. Our method allows us to span the length scales from single crystal to that appropriate to obtain polycrystal properties. The single crystal free energy coefficients can be determined from microscopic calculations (such as electronic structure and molecular dynamics) and/or available experimental structural, phonon and thermodynamic data. We simulate the microstructure and determine the stress-strain response of the polycrystal and compare it with that of a single crystal. For FePd parameters we find that the recoverable strain for a polycrystal is $\sim 40 \%$ of that for a single crystal. The polycrystal information can, in principle, serve as input to the engineering scale of calculation, where the finite element method is appropriate.
\end{abstract}

Mathematical subject classification: $74 \mathrm{~B} 20,74 \mathrm{~N} 10,74 \mathrm{~N} 15$.

Key words: martensites, shape memory alloys, polycrystals, Landau theory PACS: 81.30.Kf, 64.70. Kb, 61.72.Mm.

\section{Introduction}

There are many types of solid-solid transformations that occur in nature. A particular class of these transformations is known as martensitic transformations. Martensites are characterized by diffusionless, first-order structural phase transitions between different crystalline phases. These solid-solid transformations are important as crystal symmetry-changing phase transitions as well as for their role in inducing technologically useful materials properties. There is a related class of materials known as ferroelastics. Ferroelasticity is defined by the existence 
of two or more stable deformation states of a crystal that correspond to different arrangements of the atoms, but are structurally identical [1,2]. In addition, these deformation states are degenerate in energy in the absence of mechanical stress (e.g., there are three deformations of a tetragonal crystal resulting from a transition from a cubic crystal such as in FePd). Salient features of ferroelastic crystals include mechanical hysteresis and mechanically switchable domain patterns.

Since the ferroelastic transition (induced by either temperature change or application of stress) is normally weakly first order, or second order, it can be described to a good approximation by the Landau theory with spontaneous strain or deviation of a given ferroelastic deformation state from the parent phase as the order parameter. The strain can be coupled to intra-unit cell (shuffle) modes or other fields such as electric polarization and magnetic moment (e.g., in magnetic martensites) and thus the crystal can have more than one transition. We note that the Landau theory is a mesoscale description at a length scale on the order of one (or a few) unit cell(s). To include the inhomogeneous variation of the order parameter strain, such as across a domain wall or twin boundary, the square of the strain gradient is included in the free energy to account for the energy cost for creating a domain wall. This is refered to as the Ginzburg term and the resultant description is called the Ginzburg-Landau free energy.

There is a further subset of ferroelastic martensites (either non-elemental metals or alloy systems) that exhibit the shape memory effect [3]. These materials are characterized by highly mobile twin boundaries and (often) show precursor structures (e.g., tweed [4-6]) above the transition. Furthermore, these materials have small Bain strain, elastic shear modulus softening, and a weakly to moderately first order transition. Some representative examples include InTl, FePd, NiTi, $\mathrm{AuCd}$ and $\mathrm{CuAlZn}$. Shape memory is the ability of these materials to recover, upon heating, an apparently plastic deformation sustained below the martensitic transition temperature. To optimize the desirable functionalities of these materials it is crucial to understand microstucture such as twinning and tweed. These multiscale structures are increasingly understood to produce anomalous elastic constants [7], low-symmetry lattice vibrations, low-frequency scattering in x-ray and neutron spectra, and inhomogeneous coupling to electronic, magnetic and ferroelectric degrees of freedom. The associated multiscale dynamics 
is intimately involved in shape-memory [3], and related phenomena.

The multiscale mechanism by which local perturbations (such as defects or localized stress) can have large-scale effects on elastic patterning is anisotropic long-range strain-strain coupling. This long-range coupling arises because of elastic compatibility constraints on the allowed unit cell deformations (in the absence of bond-breaking) and is therefore indeed inherited from very local, atomic scale (i.e., microscopic), symmetries. We have recently developed models of elasticity and solid-solid phase transformations at the mesoscopic ("coarsegrained") level of a nonlinear Ginzburg-Landau description for single crystals, including the compatibility constraint self-consistently [8]. This allows a description of systematic twinning, tweed and other fine-scale hierarchical structures, i.e. to connect intrinsic multiscale phenomena in solid state physics and materials science. Our model can predict multiscale elastic textures and their consequences in a unified manner as demonstrated below through simulations.

Most commercial applications of shape memory alloys [3] (such as NiTi, FePd and $\mathrm{CuZnAl}$ ) make use of polycrystalline specimens and therefore it is important to study and compare the mechanical response of polycrystals to that of single crystals. The problem of finding the effective properties of martensitic polycrystals has been studied by analytical methods [9-11] and finite element simulations [12]. However, these methods do not account for the complex polycrystal geometry and also do not incorporate the long-range elastic interactions between the grains. Continuum simulations that span a range of length scales are good candidates to describe these issues. Recently, phase-field micro-elasticity models that employ static grains created by the Voronoi construction have been studied [13]. Nevertheless, it is important to regard the grain orientation as a thermodynamic variable since the orientation distribution (texture) of a polycrystal can change due to an applied load.

The evolution of grains during grain growth has been studied using the phasefield approach [14-16]. Although these models correctly describe the grain morphologies and domain coarsening, they do not address the issues of elasticity and crystal symmetry. A coupling of these models with continuum elasticity models of martensitic transformations provides a framework to model mechanical properties of shape memory polycrystals. Here we propose a model in which 
elastic strains are coupled to a phase-field model through an orientation field that is determined from a multi-component order parameter describing the crystal orientations. Due to this coupling, the strains as well as the grain orientations can change under an external load. Here we determine the stress-strain (constitutive) response within the coupled phase-field-elasticity model. The polycrystal response serves as a natural input for the next higher length scale, namely for the finite element methods.

\section{The Landau model for a single crystal}

The true degrees of freedom in a continuum elastic medium (such as described by Ginzburg-Landau models) are contained in the displacement field, $u(r)$, even though it is the strain fields, $\epsilon_{i j}$, which appear in the free energy [as order parameter (OP) in the anharmonic part and as non-OP in the harmonic part]. Instead of treating the strains as independent fields, one must assume that they correspond to a physical displacement field, i.e., that they are derivatives of a single continuous function. This is achieved by requiring that they satisfy a set of nontrivial compatibility relations concisely expressed as a differential relation between the strain components. Ignoring the geometrical compatibility constraint and minimizing the free energy directly would lead to the incorrect result that non-OP strain components are identically zero, and the OP strain trivially responds to perturbations, e.g. stress and local disorder, which is certainly not true. One can explicitly account for the compatibility constraint by appending it to the free energy via a Lagrange multiplier. Then the non-OP strain components can be expressed in terms of OP strain components by minimizing the free energy. This procedure results in an anisotropic, long-range interaction between the OP strain(s) as shown below.

In $d$-dimensions $(d=1,2,3)$ the displacement field has $d$ independent components at any point $x$, whereas a symmetric strain tensor nominally has $d(d+1) / 2$ independent components. Because the strain tensor is composed of derivatives of a vector field, there must be relations or constraints among its components, so that all components cannot vary in arbitrary ways. In the approximation of "geometrical linearity" these constraints are expressed by the Saint-Venant compatibility relation [17-19]. The components of the Lagrangian 


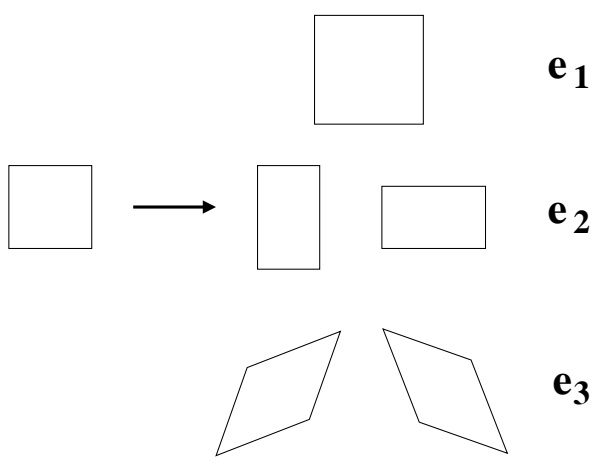

Figure 1 - The three deformations of a square lattice and associated strain tensor components: $e_{1}$ (dilatation), $e_{2}$ (deviatoric) and $e_{3}$ (shear).

strain tensor in two (or three) dimension are defined by

$$
\epsilon_{i j}=\frac{1}{2}\left(u_{i, j}+u_{j, i}+u_{k, i} u_{k, j}\right),
$$

where $u_{i, j}=\partial u_{i} / \partial x_{j}$ are the derivatives of the displacement vector with respect to material coordinates $x_{j}$ in a Cartesian frame. The last term in the above equation (with summation over $k$ implied) refers to "geometrical non-linearity" in strain and is important for finite strain deformations and lattice rotation. However, in most martensitic transitions of interest the strain is usually less than $10 \%$ and the last term can be neglected. In this approximation of geometrically linear elasticity the compatibility condition (in any dimension) is compactly written as [17-19] $\nabla \times(\nabla \times \stackrel{\leftrightarrow}{\epsilon})^{T}=0$, where $T$ denotes transpose. The zero on the right hand side indicates no source terms such as arising from dislocations or disclinations. The "incompatibility" due to such lattice topological defects can be included by using the Burgers vector (density) on the right hand side of the equation.

In one dimension there is no compatibility constraint. In two dimensions there is only one compatibility equation:

$$
\epsilon_{x x, y y}+\epsilon_{y y, x x}=2 \epsilon_{x y, x y} .
$$

There are six (two sets of three each) compatibility equations in three dimensions. In order to identify the components of the strain tensor that serve as the primary 
OP in a structural transition, symmetry methods come very handy (particularly in three dimensions). Strain corresponds to the center $k=0$ of the reciprocal lattice unit cell (i.e. in Fourier space). That is, it is a variable which does not break translation symmetry of the lattice. For a square lattice we find three symmetryadapted strains $[8,20]$, namely dilatation $\left(e_{1}\right)$, deviatoric or rectangular $\left(e_{2}\right)$ and shear $\left(e_{3}\right)$ strains defined as:

$$
\begin{aligned}
& e_{1}=\frac{1}{\sqrt{2}}\left(\epsilon_{x x}+\epsilon_{y y}\right), \\
& e_{2}=\frac{1}{\sqrt{2}}\left(\epsilon_{x x}-\epsilon_{y y}\right), \\
& e_{3}=\epsilon_{x y},
\end{aligned}
$$

which are depicted in Fig. 1.

The compatibility condition in these variables is

$$
\nabla^{2} e_{1}(r)-\sqrt{8} \nabla_{x} \nabla_{y} e_{3}(r)=\left(\nabla_{x}^{2}-\nabla_{y}^{2}\right) e_{2}(r)
$$

In Fourier space, with periodic boundary conditions, it reads

$$
k^{2} e_{1}(\vec{k})-\sqrt{8} k_{x} k_{y} e_{3}(\vec{k})=\left(k_{x}^{2}-k_{y}^{2}\right) e_{2}(\vec{k}),
$$

where $\vec{k}=\left(k_{x}, k_{y}\right)$ is the wavevector. The free energy functional for the square to rectangle transition is given by

$$
F=\int d \vec{r}\left[f_{\text {elastic }}+f_{\text {load }}\right]
$$

where

$$
f_{\text {elastic }}=\frac{A_{1}}{2} e_{1}^{2}+\frac{A_{3}}{2} e_{3}^{2}+f_{\text {local }}\left(e_{2}\right)+\frac{K_{2}}{2}\left(\nabla e_{2}\right)^{2} .
$$

Since $e_{2}$ is the order parameter for this transition,

$$
f_{\text {local }}\left(e_{2}\right)=\frac{A_{2}}{2} e_{2}^{2}+\frac{B}{4} e_{2}^{4}+\frac{C}{6} e_{2}^{6} .
$$

Here $A_{1}, A_{2}$ and $A_{3}$ denote the bulk modulus, deviatoric modulus and the shear modulus, respectively, and are a combination of second order elastic constants. 
$A_{2}$ varies linearly with temperature near the transition. The parameters $B$ and $C$ are related to higher order elastic constants and $K_{2}$ is the strain gradient coefficient and determines the energy cost of creating a domain wall (i.e., twin boundary).

These free energy parameters can be determined from the thermodynamic, structural and vibrational experimental data for a material. However, microscopic modeling such as electronic structure calculations and molecular dynamics simulations (based on atomistic potentials) can be used to determine the parameters. This provides a direct bridge between the microscopic length scale and the mesoscopic Ginzburg-Landau length scale.

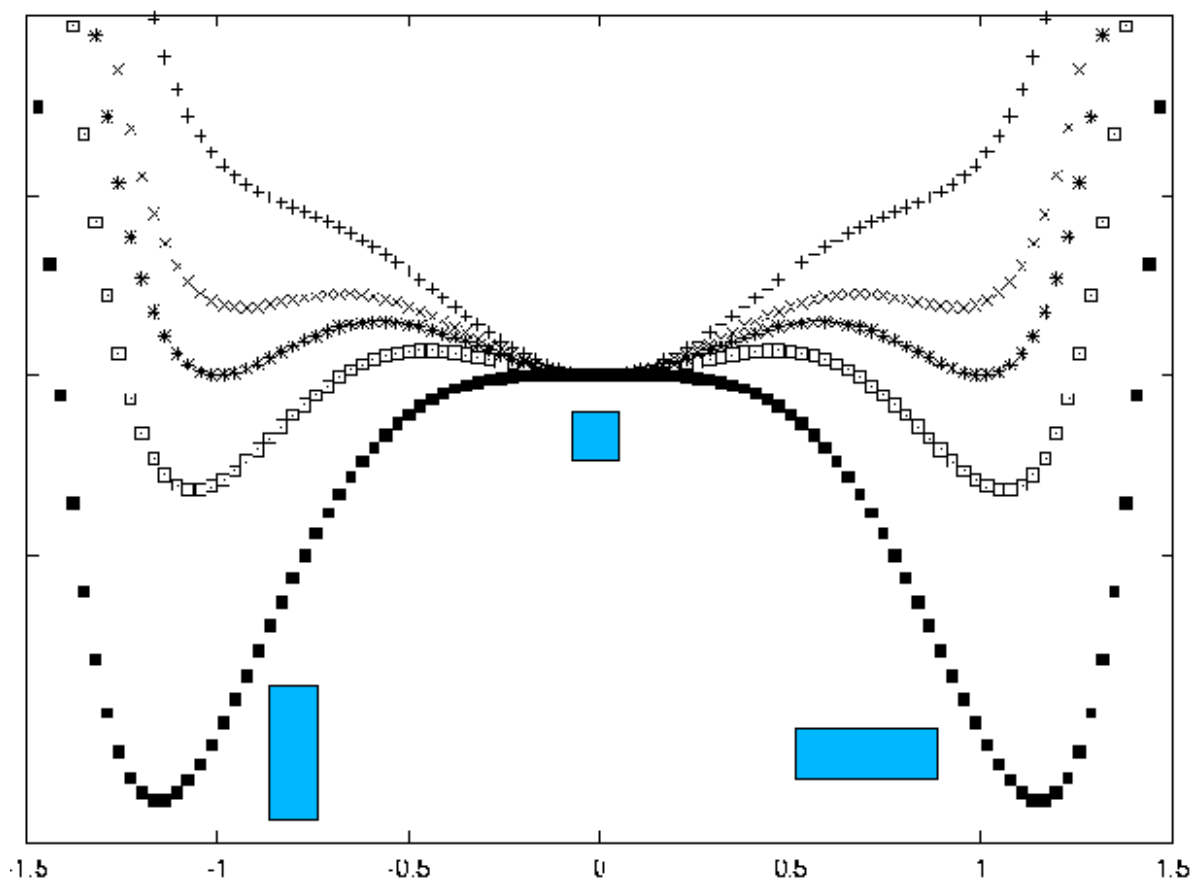

Deviatoric strain

Figure 2 - The triple well, local Landau free energy as a function of temperature.

In Fig. 2 we show the variation of the local part of the Landau free energy, $f_{\text {local }}\left(e_{2}\right)$, as a function of temperature. Above the transition temperature there is only one global minimum at zero strain corresponding to the square lattice. 
As the temperature is decreased two (symmetric) local minima appear at finite strain value. At the critical temperature there are three degenerate minima, i.e. the square lattice and the two rectangular lattices have the same energy. This is the first order transition to the martensite phase. Below this temperature the central minimum first becomes a local minimum and then a local maximum and the square lattice phase disappears. Only the two degenerate minima at finite strain corresponding to the two orientations of the rectangular lattice exist. Thus there will be a twinning between these orientation states.

If we eliminate $e_{1}$ using the compatibility constraint $[8,21]$, the harmonic energy contribution due to compression and shear strains is given by

$$
\begin{aligned}
F_{c s} & =\int d \vec{r}\left[\frac{A_{1}}{2} e_{1}(\vec{r})^{2}+\frac{A_{3}}{2} e_{3}(\vec{r})^{2}\right] \\
& =\int d \vec{k}\left[\frac{A_{3}}{2}\left|e_{3}(\vec{k})\right|^{2}+\frac{A_{1}}{2}\left|C_{2}(\vec{k}) e_{2}(\vec{k})+C_{3}(\vec{k}) e_{3}(\vec{k})\right|^{2}\right],
\end{aligned}
$$

where

$$
C_{2}=\frac{k_{x}^{2}-k_{y}^{2}}{k_{x}^{2}+k_{y}^{2}} \quad C_{3}=\frac{\sqrt{8} k_{x} k_{y}}{k_{x}^{2}+k_{y}^{2}},
$$

implying that $k_{x}= \pm k_{y}$ would minimize the elastic energy. Thus, domain walls will prefer an orientation of $\pm \pi / 4$. Equation (8) represents an anisotropic long-range interaction between the $e_{2}$ and $e_{3}$ strains. A uniaxial external stress $\sigma$ (applied along the $x$-axis) couples to strain as follows:

$$
f_{\text {load }}=-\sigma \epsilon_{x x}=-\frac{\sigma}{\sqrt{2}}\left(e_{1}+e_{2}\right) .
$$

\section{Pseudoelastic behavior}

We first illustrate through an example the use of the local free energy discussed in the previous section. We can understand the pseudoelastic behavior (i.e. mechanical hysteresis but no residual strain when the high temperature austenite phase is subjected to an external stress) based on the triple well, local Landau free energy for a single crystal. In the absence of external stress the free energy is symmetric and has a global minimum at zero strain and an inflection point at a finite strain. As an external stress is applied (it couples linearly to strain), 
the free energy becomes asymmetric and the global minimum starts shifting to finite strain values (Fig. 3, left panel). This corresponds to an (almost) linear increase in the stress-strain curve (Fig. 3, right panel). With increasing stress the inflection point first becomes a local minimum (at a finite strain) and then at a critical stress it becomes degenerate with the previously global minimum and eventually becomes the global minimum. This corresponds to the plateau region in the stress-strain curve.

\section{Free energy minima ( loading): pseudoelastic}
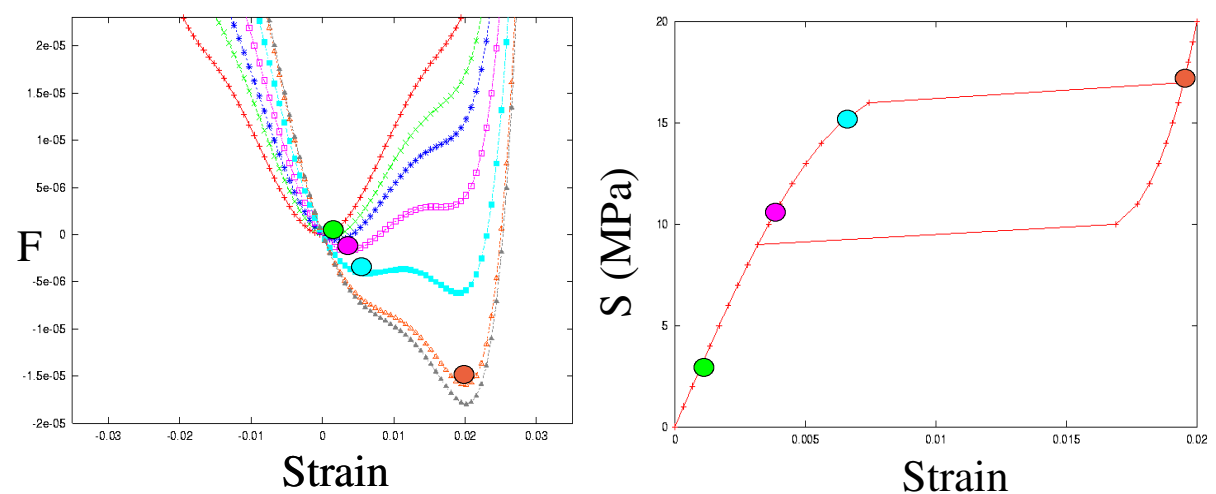

Figure 3 - Variation of the local free energy (above the martensitic transition temperature) as a function of applied load (left panel). Stress-strain response showing a pseudoelastic behavior (right panel).

If we now start unloading the crystal, the global minimum starts becoming shallower. This corresponds to the (almost) linear decrease in the stress-strain curve. At certain stress the global minimum becomes degenerate with the other minimum (near zero strain). This is related to the lower plateau in the hysteresis curve. Upon further unloading this minimum becomes local and eventually an inflection point. This is related to the linear decrease toward zero strain in the stress-strain curve. Thus, there is no residual strain despite a hysteretic loading-unloading cycle. Indeed, the simple free energy is able to capture the pseudoelastic behavior above the martensitic temperature. 


\section{The Landau model for a polycrystal}

The free energy functional for a polycrystal is written as $F=F_{\text {grain }}+F_{\text {elastic }}+$ $F_{\text {load }}$, where $F_{\text {grain }}$ is the free energy density due to the orientational degrees of freedom of the polycrystal, $F_{\text {elastic }}$ represents the elastic free energy and $F_{\text {load }}$ is the free energy contribution due to an external applied load. The polycrystalline system is described by a set of $Q$ non-conserved order parameters [15] $\left(\eta_{1}, \eta_{2}, \ldots, \eta_{Q}\right)$. In a grain growth process the grains can grow at the expense of each other and thus the order parameter is not conserved. A given grain orientation corresponds to one of the $Q$ order parameters being positive nonzero while the rest are zero. The free energy $F_{\text {grain }}$ is given by

$$
\begin{aligned}
F_{\text {grain }}= & \int d \vec{r}\left\{\sum_{i=1}^{Q}\left[\frac{a_{1}}{2} \eta_{i}^{2}+\frac{a_{2}}{3} \eta_{i}^{3}+\frac{a_{3}}{4} \eta_{i}^{4}\right]\right. \\
& \left.+\frac{a_{4}}{2} \sum_{i=1}^{Q} \sum_{j>i}^{Q} \eta_{i}^{2} \eta_{j}^{2}+\sum_{i=1}^{Q} \frac{K}{2}\left(\nabla \eta_{i}\right)^{2}\right\} .
\end{aligned}
$$

For $a_{1}, a_{2}<0$ and $a_{3}, a_{4}>0$, the first two terms in equation (11) describe a potential with $Q$ degenerate minima corresponding to $Q$ grain orientations. The gradient energy $(K>0)$ represents the energy cost of creating a grain boundary. It is also possible to associate an orientational field $\theta(\vec{\eta}, \vec{r})$ with $Q$ orientations between 0 and $\theta_{m}$, where

$$
\theta(\vec{\eta}, \vec{r})=\frac{\theta_{m}}{Q-1}\left[\frac{\sum_{i=1}^{Q} i \eta_{i}}{\sum_{i=1}^{Q} \eta_{i}}-1\right] .
$$

Thus, there are $Q$ orientations between 0 and a maximum angle $\theta_{m}$. As an example, for the $Q=5$ case used in simulations below the five minima at $\left(\eta_{0}, 0,0,0,0\right),\left(0, \eta_{0}, 0,0,0\right),\left(0,0, \eta_{0}, 0,0\right),\left(0,0,0, \eta_{0}, 0\right)$ and $\left(0,0,0,0, \eta_{0}\right)$ correspond to $\theta=0^{\circ}, \theta=\theta_{m} / 4, \theta=\theta_{m} / 2, \theta=3 \theta_{m} / 4$ and $\theta=\theta_{m}$. For the elastic free energy the linearized strain tensor in a global reference frame is defined by $\epsilon_{i j}=\left(u_{i, j}+u_{j, i}\right) / 2(i=1,2 ; j=1,2)$, where $u_{i}$ represents $i$ th component of the displacement vector and $u_{i, j}$ is its $j$ th displacement gradient.

For illustration, we again consider a two dimensional square lattice and use the symmetry-adapted linear combinations of the strain tensor defined as $[8,20]$ 
$\epsilon_{1}=\left(\epsilon_{x x}+\epsilon_{y y}\right) / \sqrt{2}, \epsilon_{2}=\left(\epsilon_{x x}-\epsilon_{y y}\right) / \sqrt{2}$ and $\epsilon_{3}=\epsilon_{x y}$. To generalize this theory for a polycrystal, the strain tensor in a rotated frame is calculated as $R(\theta(\vec{\eta})) \stackrel{\leftrightarrow}{\epsilon} R^{T}(\theta(\vec{\eta}))$, where $R(\theta(\vec{\eta}))$ is a rotation matrix. Using this transformation, the elastic free energy for a square to rectangular transition (with $e_{2}$ as the elastic order parameter) in a global frame of reference is

$$
F_{\text {elastic }}=\int d \vec{r}\left\{\frac{A_{1}}{2} e_{1}^{2}+\frac{A_{2}}{2} e_{2}^{2}+\frac{A_{3}}{2} e_{3}^{2}+f_{n l}\left(e_{2}\right)+\frac{K_{2}}{2}\left(\nabla e_{2}\right)^{2}\right\},
$$

where $e_{1}, e_{2}, e_{3}$ are defined as

$$
\begin{aligned}
& e_{1}=\epsilon_{1} \\
& e_{2}=\epsilon_{2} \cos [2 \theta(\vec{\eta})]+\sqrt{2} \epsilon_{3} \sin [2 \theta(\vec{\eta})], \\
& e_{3}=-(1 / \sqrt{2}) \epsilon_{2} \sin [2 \theta(\vec{\eta})]+\epsilon_{3} \cos [2 \theta(\vec{\eta})] .
\end{aligned}
$$

The orientation field $\theta(\vec{\eta})$ is determined from the minima of free energy in (11) using (12). Here $A_{1}=C_{11}+C_{12}, A_{2}=C_{11}+C_{12}$ and $A_{3}=4 C_{44}$, where $C_{11}, C_{12}$ and $C_{44}$ are the elastic constants for a crystal with square symmetry. $K_{2}$ is the appropriate gradient coefficient that in principle can be obtained from experimentally measured phonon dispersion data. The term $f_{n l}\left(e_{2}\right)=$ $\left(B e_{2}^{4}+C e_{2}^{6}\right)$ represents the nonlinear part of the elastic free energy and is crucial in describing (first order) structural phase transitions.

Here our goal is to simulate a uniaxial loading experiment. If we choose the $x$-axis to be the loading direction, the free energy contribution $\left[F_{\text {load }}=\right.$ $\left.\int d \vec{r} \sigma \epsilon_{x x}=-\int d \vec{r} \sigma(1 / \sqrt{2})\left(\epsilon_{1}+\epsilon_{2}\right)\right]$ due to the external load $(\sigma)$ is

$$
F_{\text {load }}=-\int d \vec{r} \frac{\sigma}{\sqrt{2}}\left(e_{1}+e_{2} \cos [2 \theta(\vec{\eta})]-\sqrt{2} e_{3} \sin [2 \theta(\vec{\eta})]\right) .
$$

Again, the strains $\epsilon_{1}, \epsilon_{2}$ and $\epsilon_{3}$ are not independent but satisfy a compatibility relationship [18]:

$$
\nabla^{2} \epsilon_{1}-\left(\frac{\partial^{2}}{\partial x^{2}}-\frac{\partial^{2}}{\partial y^{2}}\right) \epsilon_{2}-\sqrt{8} \frac{\partial^{2}}{\partial x \partial y} \epsilon_{3}=0
$$

Invoking the method introduced above for single crystal martensitic transformations [8], the strain $\epsilon_{1}$ may be eliminated using compatibility, to express the 
effective free energy $F_{\text {eff }}=F_{\text {elastic }}+F_{\text {load }}$ as

$$
\begin{aligned}
F_{\text {eff }}= & \frac{A_{1}}{2} \int d \vec{k}\left[C_{2}^{2}(\vec{k})\left|\Gamma_{2}(\vec{k})\right|^{2}+C_{3}^{2}(\vec{k})\left|\Gamma_{3}(\vec{k})\right|^{2}\right. \\
& \left.+C_{2}(\vec{k}) C_{3}(\vec{k})\left[\Gamma_{3}(\vec{k}) \Gamma_{2}(-\vec{k})+\Gamma_{3}(-\vec{k}) \Gamma_{2}(\vec{k})\right]\right] \\
& +\int d \vec{r}\left[\frac{A_{2}}{2} e_{2}^{2}+\frac{A_{3}}{2} e_{3}^{2}+f_{n l}\left(e_{2}\right)+\frac{K_{2}}{2}\left(\nabla e_{2}\right)^{2}\right. \\
& -\frac{\sigma}{\sqrt{2}}\left(e_{2} \cos [2 \theta(\vec{\eta})]-\sqrt{2} e_{3} \sin [2 \theta(\vec{\eta})]\right],
\end{aligned}
$$

where $\Gamma_{2}(\vec{k}), \Gamma_{3}(\vec{k})$ represent Fourier transforms of

$$
e_{2} \cos [2 \theta(\vec{\eta})]-\sqrt{2} e_{3} \sin [2 \theta(\vec{\eta})] \quad \text { and } \quad e_{2} \sin [2 \theta(\vec{\eta})] / \sqrt{2}+e_{3} \cos [2 \theta(\vec{\eta})]
$$

respectively, $C_{2}(\vec{k})=\left(k_{x}^{2}-k_{y}^{2}\right) /\left(k_{x}^{2}+k_{y}^{2}\right)$ and $C_{3}(\vec{k})=\sqrt{8} k_{x} k_{y} /\left(k_{x}^{2}+k_{y}^{2}\right)$. The long-range terms ensure that compatibility is satisfied within the grains as well as at the grain boundaries.

The dynamics of the grains is given by $Q$ equations

$$
\frac{\partial \eta_{i}}{\partial t}=-\gamma_{\eta} \frac{\delta F}{\delta \eta_{i}},
$$

where $\gamma_{\eta}$ is a dissipation coefficient and $i=1, \ldots, Q$, correspond to $Q$ grain orientations. The corresponding overdamped dynamics for the strains is

$$
\frac{\partial e_{2}}{\partial t}=-\gamma_{2}\left[\frac{\delta F}{\delta e_{2}}\right], \quad \frac{\partial e_{3}}{\partial t}=-\gamma_{3}\left[\frac{\partial F}{\delta e_{3}}\right],
$$

where $\gamma_{2}$ and $\gamma_{3}$ are the appropriate dissipation coefficients for the strains and $F=F_{\text {grain }}+F_{\text {eff }}$ is the total free energy of the polycrystal.

\section{Simulated microstructure and constitutive response}

We choose FePd parameters [21] for which $A_{1}=140 \mathrm{GPa}, A_{3}=280 \mathrm{GPa}$, $B=-1.7 \times 10^{4} \mathrm{GPa}$ and $C=3 \times 10^{7} \mathrm{GPa}$. FePd undergoes a face-centered cubic to face-centered tetragonal martensitic transition around $\sim 265 \mathrm{~K}$. In two dimensions this transition can be mimicked by the square to rectangle transition 
modeled above. The temperature dependent elastic constant $A_{2}$ undergoes a softening and hence controls the square to rectangle transformation. Muto et al. [22] have measured the elastic constants of FePd as a function of temperature. For the parameters in $f_{\text {grain }}$ we choose (for illustrative purposes) $a_{1}=-10 \mathrm{GPa}$, $a_{2}=-10 \mathrm{GPa}, a_{3}=10 \mathrm{GPa}, a_{4}=20 \mathrm{GPa}, Q=5$ (i.e., five different grain orientations) and $\theta_{m}=30^{\circ}$. Here, we also need to specify the grain boundary energy coefficient $K$ and the strain gradient coefficient $K_{2}$. For FePd, the strain gradient coefficient [21] has been measured to be $K_{2} / a_{0}^{2}=25 \mathrm{GPa}$, where $a_{0}$ is the lattice spacing of the crystal. The grain boundary energy coefficient is chosen as $K / a_{0}^{2}=10^{14} \mathrm{GPa}$. The lengths are scaled by $\vec{r}=\left(100 a_{0}\right) \vec{\zeta}$. For a homogeneous single crystal, using these parameter values, the free energy in equation (11) has 5 degenerate minima defined by $\theta_{0}(\vec{\eta})=0^{\circ}, 7.5^{\circ}, 15^{\circ}, 22.5^{\circ}, 30^{\circ}$, corresponding to five grain orientations. Equations (17) and (18) are solved numerically to simulate the domain structures and mechanical properties. For simplicity, we assume $\gamma_{\eta}=\gamma_{1}=\gamma_{2}=\gamma$ and use rescaled time defined by $t^{*}=t\left(10^{10} \gamma\right)$.

An initial polycrystalline configuration is first generated by solving equations (17) and (18) for a $12800 a_{0} \times 12800 a_{0}$ system with periodic boundary conditions, starting from random initial conditions. A grain growth process is simulated with $\sigma=0$ in the austenite phase so that all components of the strain tensor vanish. Grains with the above five orientations $\theta_{0}(\vec{\eta})$ form and start coarsening. We arrest the system in a given polycrystalline configuration by abruptly changing the value of the parameter $a_{1}$ from $-10 \mathrm{GPa}$ to $-160 \mathrm{GPa}$ (the parameter $A_{2}$ is also changed so that the system is in the desired phase). This sudden decrease in $a_{1}$ increases the free energy barriers between the crystalline states and the growth stops. We consider the case $A_{2}=-2 \mathrm{GPa}$. For a homogeneous system, the local part of the transformation free energy $f_{\text {elastic }}\left(e_{2}\right)$ for $A_{2}=-2 \mathrm{GPa}$ has a local maximum at $e_{2}=0$ and two degenerate global minima. In the absence of applied stress $(\sigma=0)$, the arrested polycrystal evolves into a domain pattern of the variants of the martensitic phase (there is no austenite present since $e_{2}=0$ is unstable). The strains in each grain as well as the orientation of the martensitic domain walls (i.e. twin boundaries) are determined by the orientation of the grain. This behavior is clear from (the left panels of) Fig. 4 that shows the distribution of the strain $\epsilon_{2}(\vec{r})$ (deviatoric strain relative to the global 
frame of reference). We do not show the corresponding local orientation field $\theta(\vec{\eta}(\vec{r}))$. The domain walls are oriented at angles $\theta(\vec{\eta})+\pi / 4$ or $\theta(\vec{\eta})-\pi / 4$. The average strains for this configuration are very small and correspond to a system with no macroscopic deformation. The single crystal microstructure is shown in the right panels (a), (b), (d) and (f).
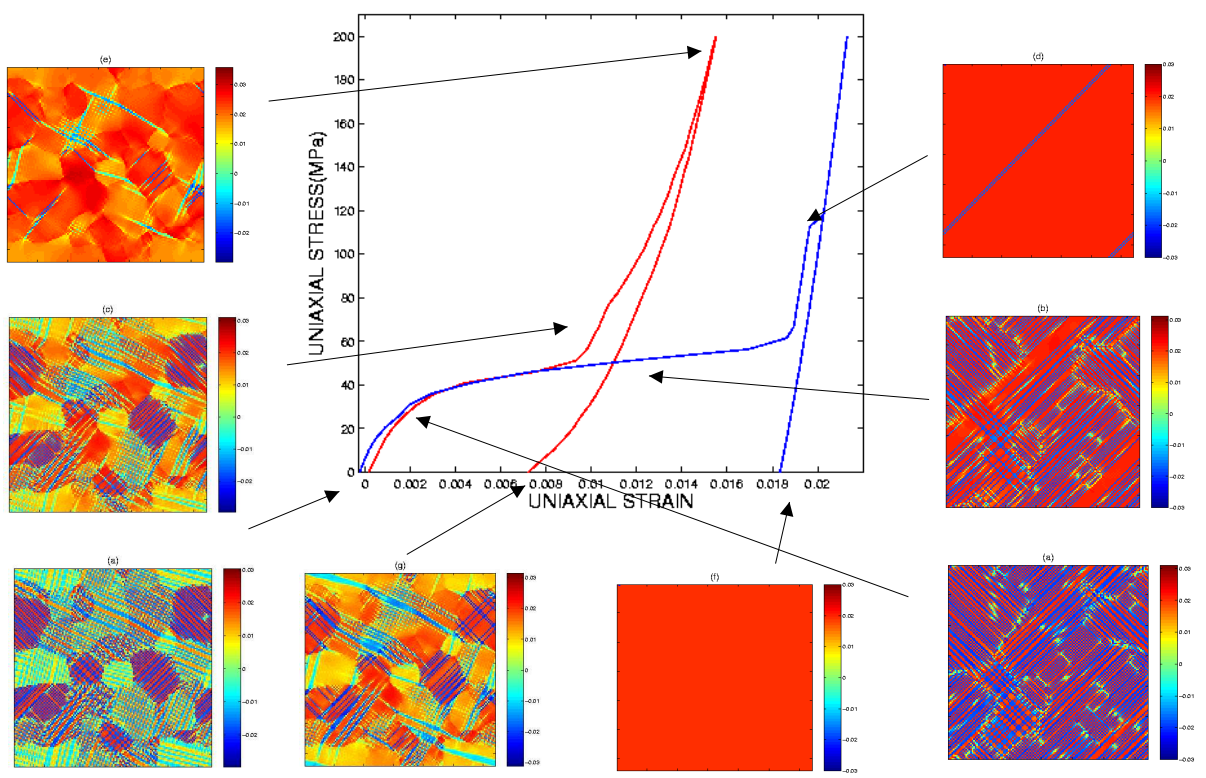

Figure 4 - Stress-strain response for the loading-uncloading cycle for a single crystal (right curve) and a polycrystal (left curve). The associated microstructure at various points during the loading cycle is also shown.

To simulate mechanical loading, an external tensile stress $\sigma$ is applied quasistatically, i.e., starting from the unstressed polycrystal configuration of left panel (a), the applied stress $\sigma$ is increased in steps of 5.13 MPa, after allowing the configurations to relax for $t^{*}=25$ time steps after each increment. The loading is continued till a maximum stress of $\sigma=200 \mathrm{MPa}$ is reached in the left panel (e). Thereafter, the system is unloaded by decreasing $\sigma$ to zero at the same rate at which it was loaded; see the left panel (g). Panel (c) relates to a stress level of $\sigma=46.15 \mathrm{MPa}$ during the loading process. The favored variants (lighter domains in the left panel) have started to grow at the expense of the unfavored variants (darker domains in the left panel). The orientation distribution $\theta(\vec{\eta}(\vec{r}))$ 
does not change much. As the stress level is increased further, the favored variants grow. Even at the maximum stress of $200 \mathrm{MPa}$, some unfavored variants persist, as is clear from panel (e).

We note that the grains with large misorientation with the loading direction rotate. Grains with lower misorientation do not undergo significant rotation. The mechanism of this rotation is the tendency of the system to maximize the transformation strain in the direction of loading so that the total free energy is minimized. Within the grains that rotate, sub-grain bands with slightly higher values of the orientation $\theta(\vec{\eta}(\vec{r}))$ are present. These bands correspond to the unfavored strain variants that still survive. Panel (g) depicts the situation after unloading to $\sigma=0$. Upon removing the load, a domain structure is nucleated again due to the local strains at the grain boundaries and the surviving unfavored variants in the loaded polycrystal configuration in panel (e). This domain structure is not the same as that prior to loading, see panel (a), and thus there is an underlying hysteresis. The unloaded configuration has non-zero average strain. This average strain is recovered by heating to the austenite phase, as per the shape memory effect. The corresponding orientation distribution (not shown) reverts to its preloading state as the grains rotate back when the load is removed.

We compare the above mechanical behavior of the polycrystal to the corresponding single crystal (right panels and the right stress-strain curve). The residual strain for the polycrystal $(\sim 0.7 \%)$ is smaller than that for the single crystal $(\sim 1.8 \%)$ due to nucleation of domains at grain boundaries upon unloading. In addition, the transformation in the stress-strain curve for the polycrystal is not abrupt because the response of the polycrystal is averaged over all grain orientations.

\section{Conclusion}

We have proposed a multiscale, Ginzburg-Landau model framework to study the constitutive response of shape memory single crystals as well as polycrystals. We discussed the role of elastic compatibility constraint and the anisotropic long-range interaction it leads to within the strain based formalism. The simple triple well, local free energy has been shown to lead to a pseudoelastic behavior. As an illustrative example, we have coupled the elastic free energy for a 
square lattice to rectangle lattice transition to a phase-field model describing crystal orientations. We studied mechanical properties of shape memory polycrystals and compared it with a single crystal. There are significant differences between the mechanical response of single crystals and polycrystals. Since the mechanical properties of the polycrystal are an average of individual grains, the stress-strain curves are smoother compared to those of the single crystals. The inhomogeneities in the polycrystal ensure that domain walls influence the mechanical behavior throughout the loading-unloading process. In the temperature regimes with nonzero residual strain, the unloaded polycrystals not only have reduced strain but also show domain microstructure. In contrast, the simulated defect free single crystals exhibit no such patterns after unloading and therefore have much higher residual strains. This finding is consistent with the fact that polycrystals have poor shape memory properties in comparison to single crystals $[9,23,24]$. We also note that measurements of texture evolution upon loading in a shape memory alloy (namely NiTi) have been recently reported [25]. Finally, we emphasize that the mesoscopic Ginzburg-Landau model provides a link with the microscopic length scales through the free energy expansion parameters and a link with the macroscopic length scales via the polycrystal constitutive response.

\section{Acknowledgements}

We acknowledge fruitful discussions with F.L. Addessio, K.H. Ahn, A.R. Bishop, K.Ø. Rasmussen, S.R. Shenoy and T.O. Williams. This work was supported by the U.S. Department of Energy.

\section{REFERENCES}

[1] V.K. Wadhawan, Introduction to Ferroic Materials (Gordon and Breach, Amsterdam, 2000).

[2] E.K.H. Salje, Phase Transformations in Ferroelastic and Co-elastic Solids (Cambridge University Press, Cambridge, U.K., 1990).

[3] K. Otsuka and C.M. Wayman (eds.), Shape Memory Materials, Cambridge University Press, Cambridge, UK (1998); MRS Bull. 27, Feb. (2002).

[4] G.R. Barsch, J.A. Krumhansl, L.E. Tanner and M. Wuttig, Scripta Metall. 21 (1987), 1257 and references therein.

[5] J.A. Krumhansl, in Lattice Effects in High-Tc Superconductors, eds. Y. Bar-Yam, T. Egami, J. Mustre de Leon, and A.R. Bishop (World Scientific, Singapore, 1992). 
[6] S.M. Shapiro, B.X. Yang, Y. Noda, L.E. Tanner and D. Schryvers, Phys. Rev. B 44 (1991), 9301.

[7] A. Planes and L. Mañosa, Solid State Phys. 55 (2001), 159.

[8] S.R. Shenoy, T. Lookman, A. Saxena and A.R. Bishop, Phys. Rev. B 60 (1999), R12537; T. Lookman, S.R. Shenoy, K.Ø. Rasmussen, A. Saxena and A.R. Bishop, Phys. Rev. B 67 (2003), 024114.

[9] K. Bhattacharya and R.V. Kohn, Arch. Rational Mech. Anal. 139 (1997), 99; Y.C. Shu and K. Bhattacharya, Acta Mater. 46 (1998), 5457.

[10] M. Huang, X. Gao and L.C. Brinson, International Journal of Plasticity 16 (2000), 1371.

[11] F. Falk, International Journal of Engineering Science 27 (1989), 277.

[12] K. Gall, T.J. Lim, D.L. McDowell, H. Sehitoglu and Y.I. Chumlyakov, International Journal of Plasticity 16 (2000), 1189.

[13] Y.M. Jin, A. Artemev and A.G. Khachaturyan, Acta Mater. 49 (2001), 2309; A. Artemev, Y.M. Jin and A.G. Khachaturyan, Philos. Mag. A 82 (2002), 1249.

[14] B. Morin, K.R.Elder, M.Sutton and M. Grant, Phys. Rev. Lett. 75 (1995), 2156.

[15] L.Q. Chen and W. Yang, Phys. Rev. B 50 (1994), 15752.

[16] J.A. Warren, W.C. Carter and R. Kobayashi, Physica (Amsterdam) 261A (1998), 159.

[17] C.L.M.H. Navier, Résumé des Leçons sur l'Application de la Mécanique, 3ème edition avec des notes et des appendices par A.J.C. Barré de Saint-Venant (Dunod, Paris, 1864).

[18] D.S. Chandrasekharaiah and L. Debnath, Continuum Mechanics (Academic, San Diego, 1996) p. 218; S. Timoshenko, History of Strength of Materials (McGraw-Hill, New York, 1953) p. 229; E.A.H. Love, A Treatise on the Mathematical Theory of Elasticity (Dover, New York, 1944); L.E. Malvern, Introduction to the Mechanics of a Continuous Medium (Prentice Hall, New Jersey, 1969).

[19] M. Baus and R. Lovett, Phys. Rev. Lett. 65 (1990), 1781; Phys. Rev. Lett. 67 (1991), 406; Phys. Rev. A 44 (1991), 1211.

[20] G.R. Barsch and J.A. Krumhansl, Phys. Rev. Lett. 53 (1984), 1069.

[21] S. Kartha, J.A. Krumhansl, J.P. Sethna and L.K. Wickham, Phys. Rev. B 52 (1995), 803.

[22] S. Muto, R. Oshima and F.E. Fujita, Acta Metallurgica et Materialia 38 (1990), 685.

[23] K. Enami, V.V. Martynov, T. Tomie, L.G. Khandros and S. Nenno, Trans. Japan Inst. Metals 22 (1981), 357.

[24] Y.D. Kim and C.M. Wayman, Metall. Trans. 23A (1992), 2981.

[25] R. Vaidyanathan, M.A.M. Bourke and D.C. Dunand, Acta Mater. 47 (1999), 3353. 